\title{
Simulation of storage performance on hydro- pneumatic driveline in dual hybrid hydraulic passenger car
}

\author{
Faizil Wasbari ${ }^{1,2, *}$, Rosli Abu Bakar ${ }^{1}$, Leong Ming Gan ${ }^{1}$, Ahmad Anas Yusof ${ }^{2}$, and Zarina \\ Amat Jafar ${ }^{1}$ \\ ${ }^{1}$ Faculty of Mechanical Engineering, Universiti Malaysia Pahang (UMP), 26600 Pekan, Pahang, \\ Malaysia \\ ${ }^{2}$ Faculty of Mechanical Engineering, Universiti Teknikal Malaysia Melaka (UTeM), Hang Tuah Jaya, \\ 76100 Durian Tunggal, Melaka, Malaysia
}

\begin{abstract}
The charging process is one of the critical processes in the hydro-pneumatic driveline storage system. It converts the kinetic energy of the vehicle braking and coasting to the compression energy. This energy is stored in the storage device called the accumulator. The system is planned to be used on the dual hydro-pneumatic hybrid driveline and applied to a hydraulic hybrid passenger car. The aim of this paper is to find the effect of charging parameters on the storage performance through simulation. Through the storage behaviour, the desirable and optimal sizing of the accumulator can be selected. The paper emphasized on the effect of pressure elevation, pre-charge pressure, effective volume, thermal reaction and required time of the accumulator's charging process. The circuit of charging process has been designed and simulated by using the hydraulic tool in the Automation Studio software. The simulation results were corroborated through the component specification for data rationality. Through the simulation, it was found that pre-charge pressure had a significant effect on the charging process. It determined the efficiency of the effective volume. The higher the pressure elevation, the higher the effective volume. Nevertheless, the more energy required to compress the nitrogen gas in the bladder. Besides, in term of volume displacement, higher volume displacement reduced charging time and lower the fluid temperature. The simulation had been positively highlighted the critical point in charging process which later on, benefited the sizing process in the component selection specification.
\end{abstract}

\section{Introduction}

Lately, hybrid technology has become so popular in the field of automotive due to the factor of energy-saving, environmentally friendly and also more efficient compared to conventional vehicles [1]. In the field of automotive, hybrid means the vehicle has more than one propulsion system to move the vehicle. There are several hybrid technology has

*Corresponding author: faizilwasbari13@gmail.com 
been developed such as hybrid electric, hybrid flywheel, hybrid hydraulic and pneumatic. However, hybrid electric is more dominant as it has been in the phase of commercialization. Based on hybridcars.com and US department of energy report on 2015, a total of 3.5 million number of the hybrid electric vehicle were sold by several manufacturers such as Toyota, Honda, Audi, Volkswagen, BMW and much more in the United States from 1999 until December 2014 [2][3]. Hybrid pneumatic and flywheel are still in the phase of research and development. Hybrid hydraulic turns popular among heavy vehicles such as buses and garbage trucks [4][5][6]. This statement is entirely realistic because it is a power density type of energy. It can drive almost all kinds of vehicles. This technology has been in the market and becomes increasingly popular in its categories. So far, innovation of hydraulic hybrid technology is focused on applying the technology to a passenger car [7]. Many research were done to ensure the success of this technology. However, it is still in the phase of research and development [8][9].

The hydraulic hybrid technology uses ICE and hydraulic motor as a propulsion while the hydro-pneumatic accumulator acts as power storage. Many types of research have been conducted related to this technology and it can be classified into four categories. There are internal combustion engine (ICE), hydro-pneumatic driveline, transmission system, and control system as illustrated in Fig. 1 [10][11][12]. The aim of this paper is to focus on the study of the hydro-pneumatic driveline. The driveline consist of five subsystems called propulsion, a regenerative system, storage, transmission, and control subsystem. The propulsion is a mechanism to move the vehicle. The regenerative is utilizing braking and coasting energy and changes them into the compression energy. The energy is then stored as potential energy in the hydro-pneumatic accumulator. The stored energy will be channelled to the second propulsion unit when the energy is required. The last subsystem is the main control system. It controls all activities in the hybrid system.

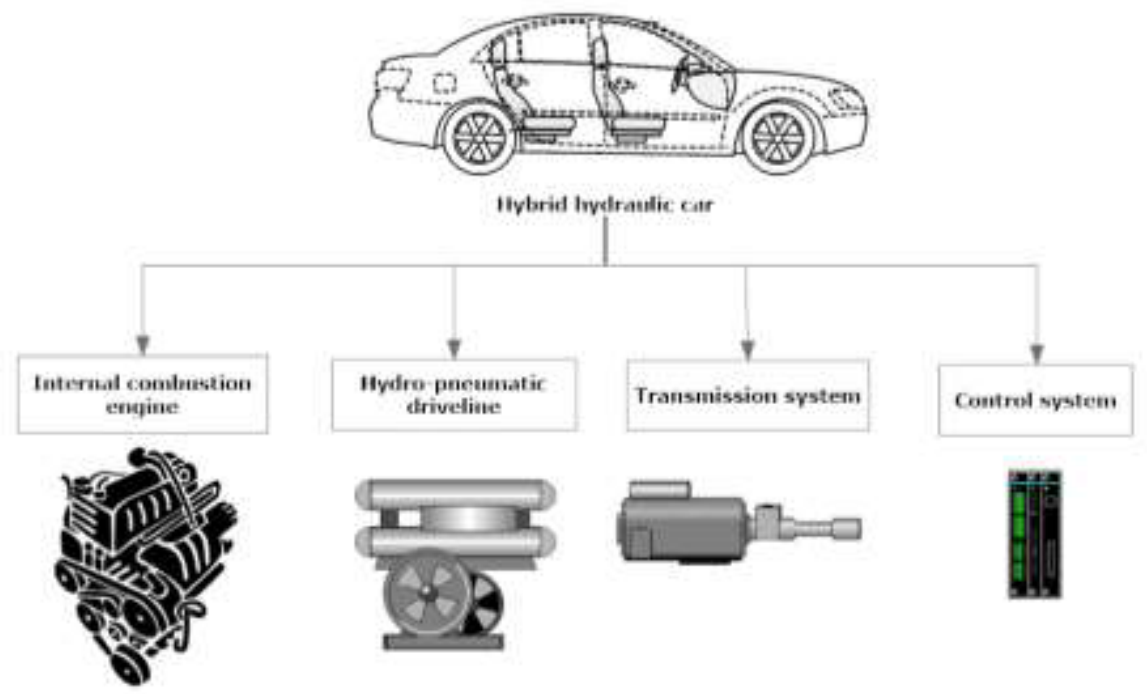

Fig. 1. Research regarding hydraulic hybrid car mostly focuses on the sub-systems breakdown.

One of the key components for hydro-pneumatic driveline is a storage system. It consists of the accumulator, pressure valve and safety block. More detail, the accumulator comprises a casing, bladder with $99.9 \%$ dry nitrogen, pressure valve and safety block. Casing design is based on the pressure vessel standard because it involves security matters. Bladder made out of an elastomer material to withstand high-pressure hydraulic oil. Nitrogen gas used as compression medium because it has a high heat absorption resistance 
to pressure changes. Air do not have endurance in such a way, and it can cause an explosion. The pressure gauge is used as an indicator of the pressure level in the accumulator. Last but not least is safety block. It acts as a pressure relief valve to protect the accumulator from the over-pressure. With this component, charging operation is safer and more reliable. Storage system carries out two main functions called charging and discharging process [13]. The charging process involves compression of hydraulic oil to the nitrogen bladder. This process creates compression energy which was then referred as potential energy. Along the compression process, the temperature changes are imperative for review. In comparison with pneumatic compression, hydro-pneumatic compression produces less heat because hydraulic oil is incompressible and also, act as a coolant to absorb the heat generated by nitrogen compression. It can be said that the system is like built-in with a heat exchanger [14].

Lammert et al. (2014) conducted lab scale experiment to parcel delivery truck. The truck used an $83.3 \mathrm{~L}$ accumulator that operated at 241 to 276 bar. He found that the configuration was able to increase $19 \%$ to $52 \%$ of fuel consumption in the diesel engine while $30 \%$ to $56 \%$ saving in the gasoline engine [15]. Kepner (2002) had been using the $54.5 \mathrm{~L}$ gas bladder accumulator on the $5.4 \mathrm{~L} \mathrm{V8}$ sports utility vehicle. The accumulator operated at 172 to 345 bar. He found that the arrangement was likewise able to cut the emission that will affect pollutions [16]. Boretti \& Zanforlin (2014) took their first step to simulate the hybrid system in passenger car application. The high-pressure accumulator operates between 135 to 485 bars and the low-pressure tank 3.5 to 13.5 bars. The accumulators have a low specific energy (1.8-4 Wh/Kg) but a wide range of specific power $(7-4,000 \mathrm{~W} / \mathrm{kg})$. They found that the system was able to achieve $30 \%$ better fuel economy [8]. The study has contributed significantly to the research for developing hydro-pneumatic hybrid driveline, but none of the studies related specifically to the subsystem. References also do not state clearly why the related pressure was used.

To answer the above statement, the Automation Studio software has been used to simulate the effect of fluid power parameters on the performance of charging process. Automation Studio is a tool for design, simulation of complex automation, documentation, and training. The software includes hydraulic, pneumatic and electrical operative devices as well as a command part diagram. It also provides technical and commercial data for simulation. Undoubtedly that the software such as Matlab has high flexibility to run a simulation that is related to the case study, but the Automation Studio has the advantage in terms of circuit design, functional simulation, fluid power component sizing, system design, validation and virtual simulation [17]. These advantages had resulted the Automation Studio more suited for this project. Currently, Automation Studio has improved one step ahead by connecting some applications with Simulink, Matlab [18]. It is hoped that more efforts will be made to improve the scope of the software application.

At the moment, many types of research focus on the simulation of the fuel consumption in the hybrid passenger car [19][20][8][21]. Nonetheless, a few only carrying out the detailed research related to the subsystem itself. Therefore, this research emphasizes on the effects of fluid power parameters to the performance of charging process on the hydropneumatic driveline. By knowing the effects of each parameter, perhaps, the process of selecting the right storage size and capacity can be conducted with more accurate. The accuracy is important because it ensures that the storage system always safe and operate at optimum levels. In the future, the hydro-pneumatic driveline is planned to be adopted on the dual hybrid hydraulic system as shown in Fig. 2. 


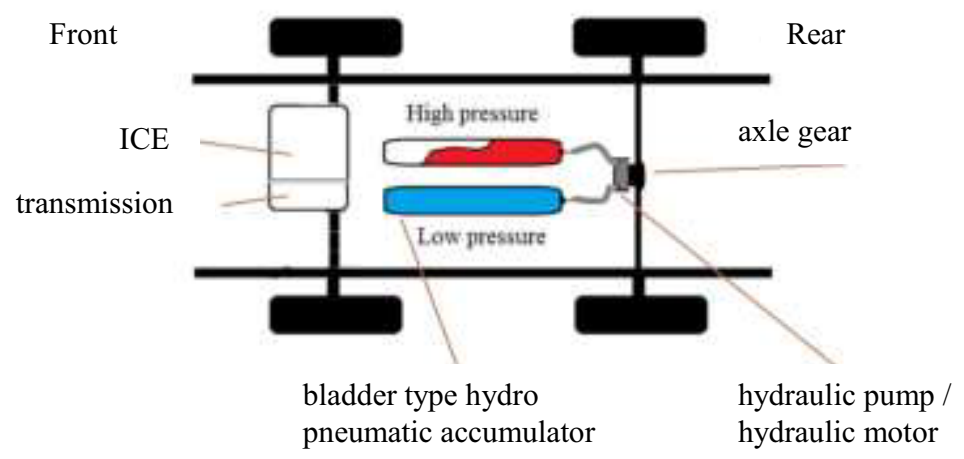

Fig. 2. Configuration of dual hybrid hydraulic car.

\section{Methodology}

The task started by designing a schematic diagram by using Automation Studio software. There are two types of simulation involve in the design which is functional and analysis simulation. Functional simulation is to ensure operation of the charging function works as desired while analysis is to get output data when all specifications are included. The analysis is divided into two. First, the internal effect of nitrogen bladder on compression and second is the effects of the schematic system to charging performance. For the internal compression effects on nitrogen bladder, the analysis made by using equations derived from Boyle-Mariotte's law for gasses [22][14], which takes into account the compressibility of the gas as illustrated in Fig. 3.

$$
\Delta V_{\text {ideal }} \leq V_{1}-V_{2}
$$

where $\Delta \mathrm{V}_{\text {ideal }}$ is the effective volume, $\mathrm{V}_{1}$ is volume before compression and $\mathrm{V}_{2}$ is volume after compression.

$$
p_{0} \times V_{0}=p_{1} \times V_{1}=p_{2} \times V_{2}
$$

where $\mathrm{p}_{0}, \mathrm{p}_{1}, \mathrm{p}_{2}$ is pressure at a certain stage of compression and $\mathrm{V}_{0}, \mathrm{~V}_{1}, \mathrm{~V}_{2}$ is the volume at a certain stage of compression. Refer to Error! Reference source not found. for detail stage of charging. However, if the condition is not in isothermal then, the equation is as follows,

$$
V_{\text {effective }}=\Delta V_{\text {ideal }} \cdot K_{i}
$$

where $\mathrm{V}_{\text {effective }}$ is effective volume, $\Delta \mathrm{V}_{\text {ideal }}$ is ideal volume under isothermal and $\mathrm{K}_{\mathrm{i}}$ is correction factor.

This is the simplified equation to calculate the average time required for the filling process. It is assumed that the flow entering the accumulator is constant and transient effect is neglected.

$$
V_{\text {effective }}=Q_{i} \cdot t_{i}
$$

where $\mathrm{V}_{\text {effective }}$ is effective volume, $\mathrm{Q}_{\mathrm{i}}$ is the flow rate and $\mathrm{t}_{\mathrm{i}}$ is the filling time. The equation (4) is then rearranged, 


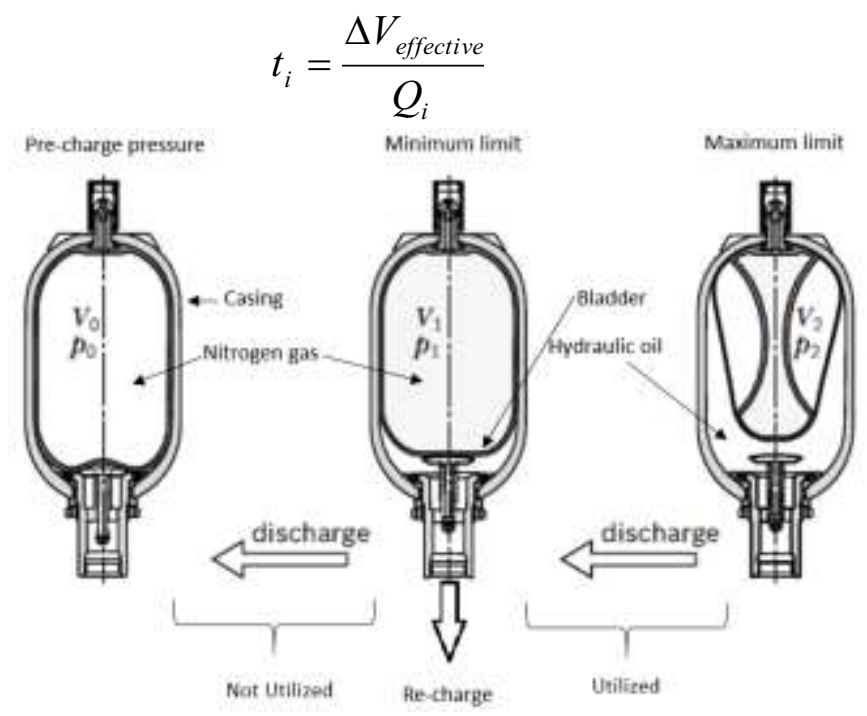

Edited source: https://www.boschrexroth.com/[14]

Fig. 3. Bladder condition during charging and discharging.

Fig. 4 shows the schematic that has been used to simulate the accumulator's process of charging. The diagram considers the flow from the pump is constant at all points of the system. Charging process starts with pumping fluid from pump to accumulator unit. In this process, the pressure change occurred. The pressure different through the pump can be expressed as follows [22],

$$
\Delta p=p_{\text {out }}-p_{\text {in }}
$$

where $\Delta p$ is the pressure different $\left(\mathrm{N} / \mathrm{m}^{2}\right), \mathrm{p}_{\text {out }}$ is pump output pressure $\left(\mathrm{N} / \mathrm{m}^{2}\right)$ and $\mathrm{p}_{\text {in }}$ is pump input pressure $\left(\mathrm{N} / \mathrm{m}^{2}\right)$. Since the main function of the pump is to create flow, the actual output flows produced by the pump can be expressed as

$$
Q_{\text {out }}=C \cdot \omega \cdot \eta_{V}
$$

where $\mathrm{Q}_{\text {out }}$ is pump output flow rate $\left(\mathrm{m}^{3} / \mathrm{s}\right), \mathrm{C}$ is displacement $\left(\mathrm{m}^{3} / \mathrm{rad}\right)$, $\omega$ is nominal speed ( $\mathrm{rad} / \mathrm{s})$ and $\eta_{\mathrm{V}}$ is volumetric efficiency. Then, equation (8) is used to calculate the shaft torque required by the pump.

$$
T=\frac{C \times \Delta p}{\eta_{M}}
$$

where $T$ is torque $(\mathrm{Nm}), \mathrm{C}$ is displacement $\left(\mathrm{m}^{3} / \mathrm{rad}\right), \Delta \mathrm{p}$ is the pressure different and $\eta_{M}$ is mechanical efficiency. Shaft power for the pump can be calculated by inserting equation (8) into equation (9).

$$
P_{S}=T_{A} \cdot \omega \cdot \eta_{\text {Overall }}
$$


where $P_{S}$ is shaft power (Watt), $T_{A}$ is torque $(\mathrm{Nm}), \omega(\mathrm{rad} / \mathrm{s})$ and $\eta_{\text {Overall }}$ is overall efficiency. Theoretical fluid power produced by the pump can be expressed as

$$
P_{P}=Q_{\text {out }} \cdot \Delta p \cdot \eta_{\text {overall }}
$$

where $P_{P}$ is pump power (Watt), $Q_{\text {out }}$ is pump output flow rate $\left(\mathrm{m}^{3} / \mathrm{s}\right), \Delta \mathrm{p}$ is the pressure different and $\eta_{\text {Overall }}$ is overall efficiency. The pressure relief valve (PRV) works as a safety valve. The pre-set pressure is the maximum pressure that is allowed to be working in the system. If the charging pressure were exceeded, the PRV would be opened to stabilize the pressure. So, the overpressure never occur.

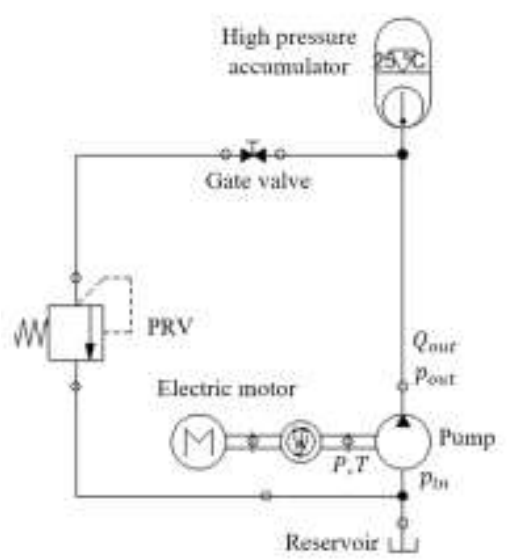

Fig. 4. Schematic diagram for charging process.

Based on the literature, the prescribed pressure is set from 0 to 400 bar while pre-charge pressure is from 10, 30 and 50 bar. If the value of pre-charge is too high, it produces the greater resistive force in the filling or charging process. This will give impact to the power to be supplied. Flow rate affects directly to the charging process, however in operation, the volume displacement is more suitable because it is associated with the specification of the pump. The combination of volume displacement and rotational speed produce flow rate. The volume displacement facilitate determination of the pump specifications including the speed and the appropriate type of pump to be used. The output is expected to present the effects of pressure elevation, pre-charge pressure and volume displacement to the storage performance which is effective volume, charging time and temperature.

\section{Simulation}

Simulation was done by using Automation Studio 6.1 software. Simulation is based on a Graphical User Interface (GUI) which is integrated with the code and equation. The equation is flexible which can be programmed by changing the root equation setting. All specifications for components, fitting, and measurement tool can be inserted into the tool's setting. Since the study involves bulk modulus and heat transfer, so the two functions were enabled. Effect of bulk modulus was considered because the present of compression process in the bladder. By enabling the bulk modulus setting, the simulation considers the effects of pressure increase respect to the volume in charging and discharging process. The thermal setting takes into account the fluid heating in contact with the pipe component inner surface. This simulation assumed that the heat transformation is isothermal where the fluid temperature remains constant. The heat exchange between gas and atmosphere is instantaneous. Pressure elevation is set as independent variable, temperature, effective 
volume and charging time as dependence variable. As for pre-charge pressure and volume displacement, the parameters are set as a control variable. For this project, the specifications used are as Table 1.

Table 1. Simulation parameters.

$\begin{array}{llll}\text { Motor rotational speed } & 2000 \mathrm{RPM} & \text { Initial liquid volume } & \text { O liter } \\ \text { Pump thermal efficiency } & 90 \% & \text { Accumulator int. diameter } & 225 \mathrm{~mm} \\ \text { Pump heat transfer coeff. } & 10 \mathrm{~W} / \mathrm{m} 2 \mathrm{~K} & \text { Type of process } & \text { Isothermal } \\ \text { Hydraulic oil } & H y d . A W-32 & \text { Gas type } & \text { Nitrogen } \\ \text { Ambient temperature } & 25^{\circ} \mathrm{C} & \text { Gas temperature } & 25^{\circ} \mathrm{C} \\ \text { Cracking pressure } & 400 \mathrm{bar} & \text { Hydraulic line } & \text { Steel } \\ \text { Port } 1\left(\mathrm{C}_{\mathrm{v}}\right) & 12 & \text { Line type } & \text { NPS } 1 / 4-D N 8\end{array}$

\section{Results and discussion}

\subsection{Effective volume}

Fig. 5, Fig. 6 and Fig. 7 have shown the effects of effective volume with regards to the pressure change for $\mathrm{V}_{\mathrm{d}}=10 \mathrm{~cm}^{3} / \mathrm{rev}$ and $\mathrm{V}_{\mathrm{d}}=50 \mathrm{~cm}^{3} / \mathrm{rev}$ at a pre-charge pressure of 10 bar, 30 bar and 50 bar. The graphs show that the effective volume has proportional relation to the pressure elevation. At 10 bar pre-charge pressure, it was found that the filling process will only start after preceding pressure of 10 bar. Initially, hydraulic oil pressure is at the level of negative (-ve) due to its level of pressure is lower than pre-charge value [14]. While the pressure increased, the hydraulic oil pressure will be on the same footing with the value of pre-charge. At this stage, the pressure difference is zero then; there is nothing happened even little compression. After the pressure exceeds the pre-charge value, the pressure difference is positive $(+v e)$, and the filling process begins. The selfsame concept also happens to pre-charge 30 bar and 50 bar. This is proved through the manual obtained from manufacturer Bosch and Parker [23][14]. Regarding bulk modulus, the nitrogen gas bulk modulus is low, so it has high compressibility. That means it has a higher level of compression maneuverability than hydraulic oil. Thus, the hydraulic oil is a medium to compress nitrogen gas in the bladder.

The effect of compression can be seen on two conditions; first, on the level below 100 bar and second, the level of effective maximum volume. At the level below 100 bar, the graph with pre-charge 10 bar experienced sharp increases from 0 to 40 liters compared to pre-charge 30 to 50 bar. This happens because of the value of the compression resistance of 10 bar is lower than 30 and 50 bar. Therefore, it is faster and more easily compressed. It can be sorted as compression resistance value of pre-charge $10 \mathrm{bar}<30 \mathrm{bar}<50$ bar. There is research found 30\% losses in stored energy [24]. The maximum effective volume level does not change when the volume displacement increase but it is becoming less when the pre-charge is increased. Manual for accumulator also supports this statement. In Fig. 6 and Fig. 7, there had been a small bump at the negative pressure different. It happens because the higher the value of pre-charge led to the higher resistance to the compression. The scenario creates a condition less-refuse between both medium and results in fluctuation. This value forms the small bump at the beginning of the graph. With 10 bar pre-charge, the average effective volume efficiency about $96 \%$. When the pre-charge was increased to 30 bar, the efficiency was reduced to $89 \%$ and at 50 bar pre-charge, the efficiency reduced to $86 \%$. 


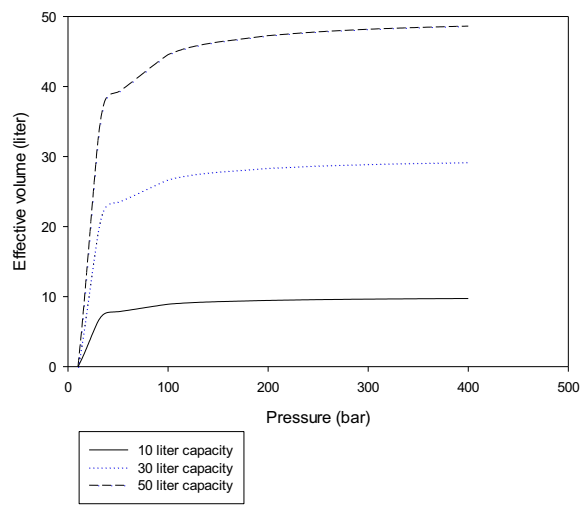

(a)

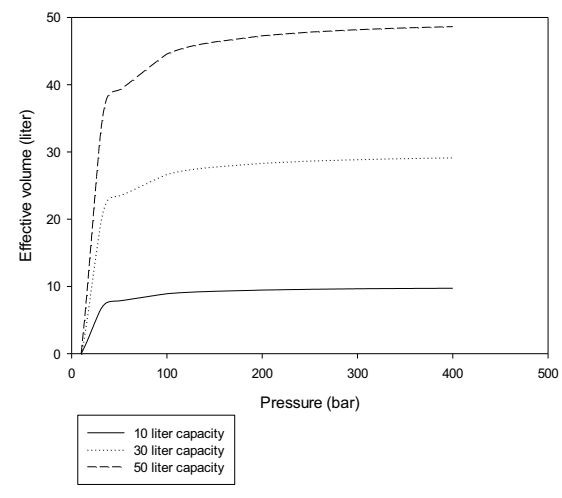

(b)

Fig. 5. Effects of effective volume (liter) with regards to pressure change for $V_{d}=10 \mathrm{~cm}^{3} / \mathrm{rev}$ (a) and $\mathrm{V}_{\mathrm{d}}=50 \mathrm{~cm}^{3} / \mathrm{rev}$ (b) at pre-charge pressure of $10 \mathrm{bar}$.

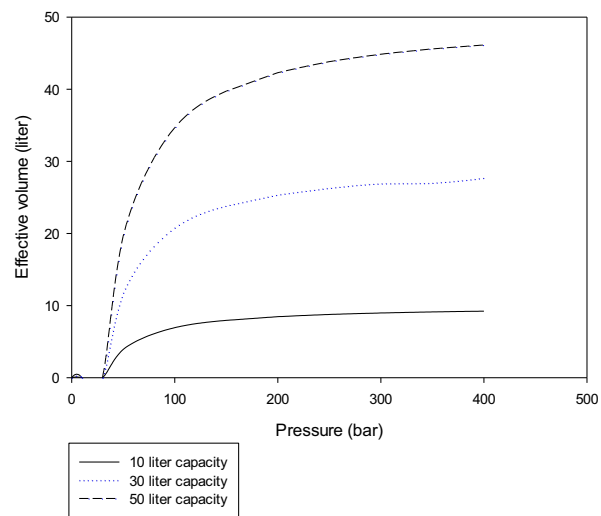

(a)

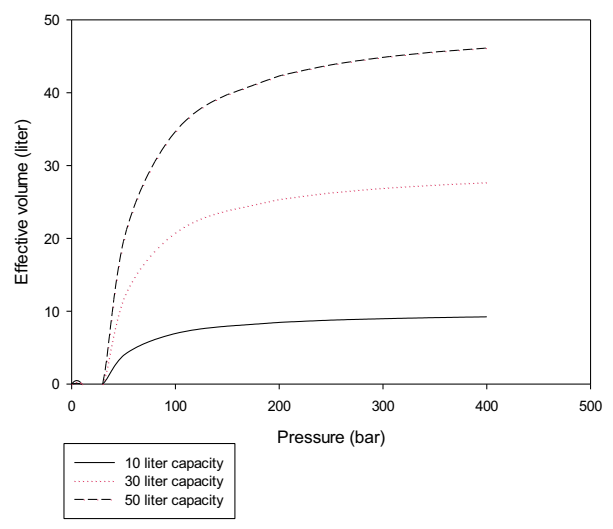

(b)

Fig. 6. Effects of effective volume (liter) with regards to pressure change for $V_{d}=10 \mathrm{~cm}^{3} / \mathrm{rev}$ (a) and $\mathrm{V}_{\mathrm{d}}=50 \mathrm{~cm}^{3} / \mathrm{rev}(\mathrm{b})$ at pre-charge pressure of $30 \mathrm{bar}$.

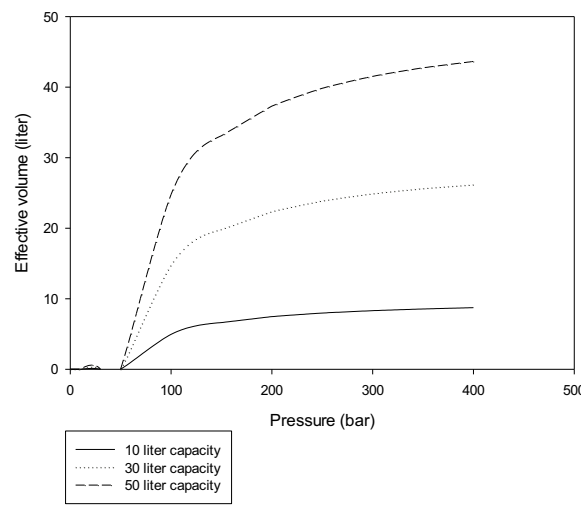

(a)

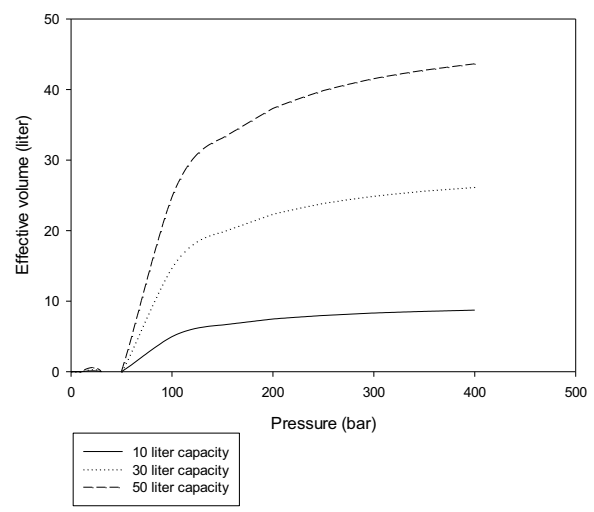

(b)

Fig. 7. Effects of effective volume (liter) with regards to pressure change for $V_{d}=10 \mathrm{~cm}^{3} / \mathrm{rev}$ (a) and $\mathrm{V}_{\mathrm{d}}=50 \mathrm{~cm}^{3} / \mathrm{rev}(\mathrm{b})$ at pre-charge pressure of $50 \mathrm{bar}$. 


\subsection{Temperature effect}

The effects of pressure elevation, pre-charge pressure and volume displacement on temperature elevation were shown in Fig. 8, Fig. 9 and Fig. 10. Overall, the temperature of volume displacement $50 \mathrm{~cm}^{3} / \mathrm{rev}$ is lower compared to volume displacement $10 \mathrm{~cm}^{3} / \mathrm{rev}$ for all pre-charge pressure 10, 30 and 50 bar. The lowest temperature is at negative (-ve) pressure different which means lower than pre-charge value and before compression happened. The supreme temperature for all case is $55^{\circ} \mathrm{C}$ at pre-charge 10,30 and 50 bar, volume displacement $10 \mathrm{~cm}^{3} / \mathrm{rev}$ and capacity 50 liters. It was about $30^{\circ} \mathrm{C}$ different from the ambient temperature. The statement that the compression process causes the rise in temperature can be proved here. Before pre-charge pressure, the temperature is at ambient and unchanged. However, when the pressure increased exceeded the pre-charge value, this is the start of the rise in temperature. Thus, the assertion that the compression process leads to the gas temperature to rise is correct and proven [24]. However, there is one situation where the occurrence of a sudden spike of temperature happened. It was at the volume displacement $50 \mathrm{~cm}^{3} / \mathrm{rev}$, pre-charge 10 bar and 10 liters capacity. Significant elevation occurred at 300 bar to 400 bar. The rise happened because of the occurrence of overcompression on the bladder. Since the bladder is usually made of elastomer, thus, it is elastic. However, it has a certain limit of elasticity which if the pressure exceeds the limits then it can damage the bladder or perhaps reduced the service life. No wonder some manufacturers limits the pressure difference to keep the bladder from over stressed, leading to excessive compression and strong gas heating.

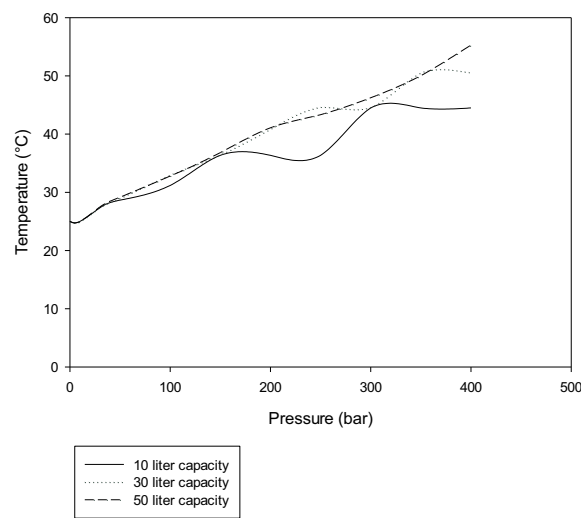

(a)

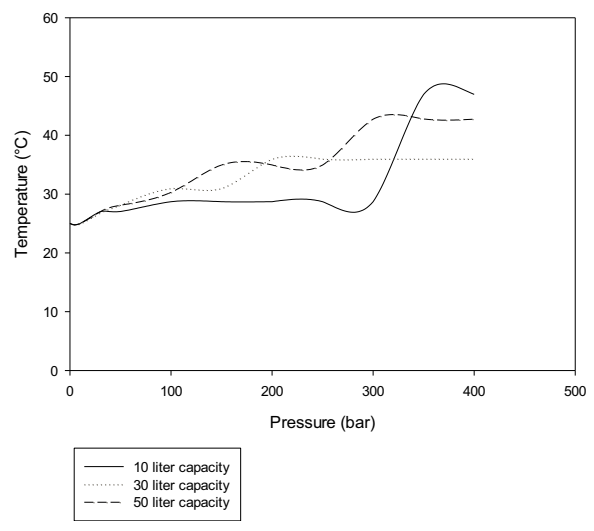

(b)

Fig. 8. Temperature elevation $\left({ }^{\circ} \mathrm{C}\right)$ for $\mathrm{V}_{\mathrm{d}}=10 \mathrm{~cm}^{3} / \mathrm{rev}(\mathrm{a})$ and $\mathrm{V}_{\mathrm{d}}=50 \mathrm{~cm}^{3} / \mathrm{rev}$ (b) at pre-charge pressure of $10 \mathrm{bar}$. 


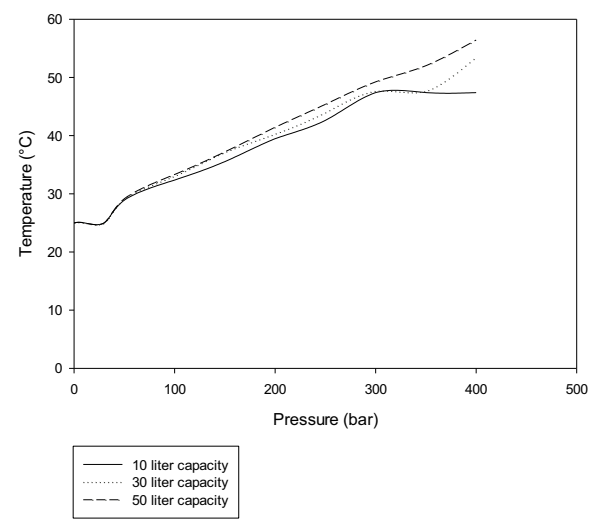

(a)

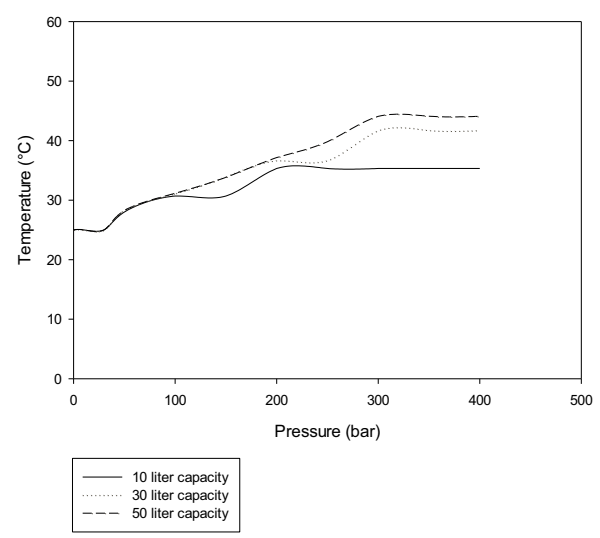

(b)

Fig. 9. Temperature elevation $\left({ }^{\circ} \mathrm{C}\right.$ ) for $\mathrm{V}_{\mathrm{d}}=10 \mathrm{~cm}^{3} / \mathrm{rev}$ (a) and $\mathrm{V}_{\mathrm{d}}=50 \mathrm{~cm}^{3} / \mathrm{rev}$ (b) at pre-charge pressure of 30 bar.

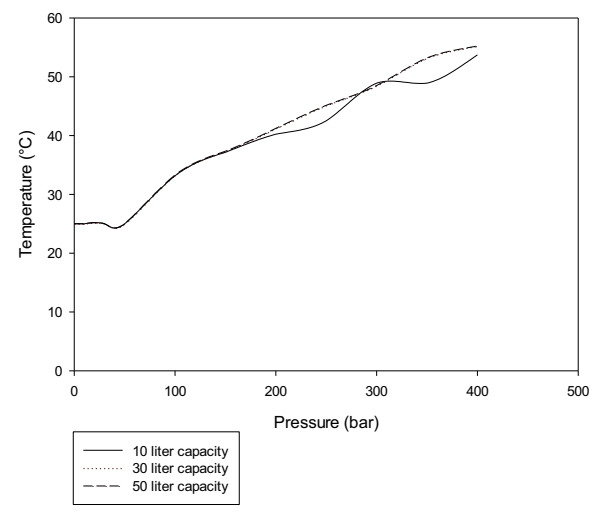

(a)

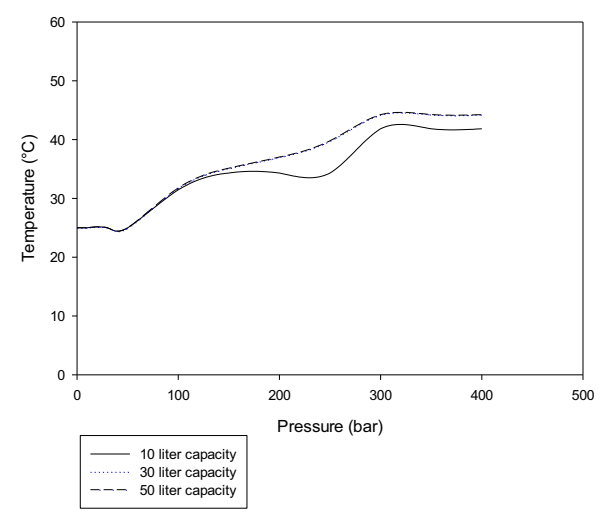

(b)

Fig. 10. Temperature elevation $\left({ }^{\circ} \mathrm{C}\right)$ for $\mathrm{V}_{\mathrm{d}}=10 \mathrm{~cm}^{3} / \mathrm{rev}$ (a) and $\mathrm{V}_{\mathrm{d}}=50 \mathrm{~cm}^{3} / \mathrm{rev}$ (b) at pre-charge pressure of 50 bar.

\subsection{Charging time}

Fig. 11, Fig. 12 and Fig. 13 have shown the effects of pressure elevation, pre-charge pressure and volume displacement on charging time. Overall, the volume displacement $\mathrm{V}_{\mathrm{d}}=$ $50 \mathrm{~cm}^{3} / \mathrm{rev}$ for each 10,30 and 50 bar pre-charge produces shortest charging time compared to $\mathrm{V}_{\mathrm{d}}=10 \mathrm{~cm}^{3} / \mathrm{rev}$. This difference is due to the flow rate factor. Pump that has a high displacement volume can fill the storage at a faster rate. Therefore, the volume displacement is imperative and needs to be considered during the selection of the pump type and capacity while performing the process of sizing. The greater the capacity of the storage, it will take longer to be filled. The graph shows the existence of negative pressure differences in each level. It happens because of the higher the value of pre-charge then the higher resistance to the compression. The scenario creates a condition less-refuse between both medium and results in fluctuation. This value forms the small bump at the beginning of the graph. The graph also shows that the charging process takes quite long to compress 0 
to 100 bar relative to 100 bar to 400 bar. This possibility is related to the characteristics of bladder elasticity and also nitrogen gas properties. The important thing that should be avoided for the charging process is the use of an unsuitable pump. If the volume displacement is small but storage capacity and pre-charge pressure are high, then the filling process took a very long time to complete as shown in Fig. 13(a).

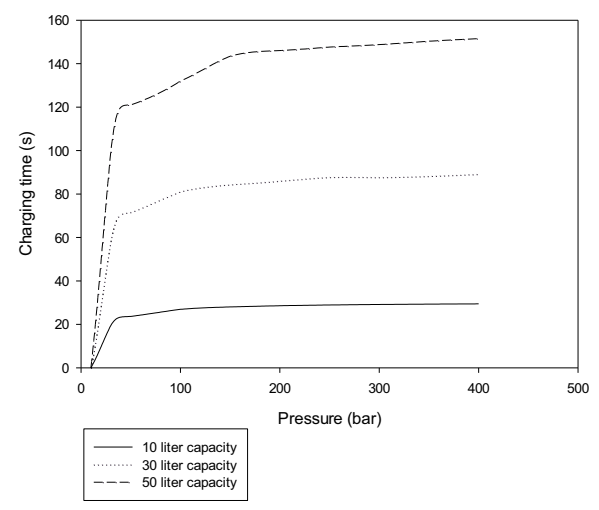

(a)

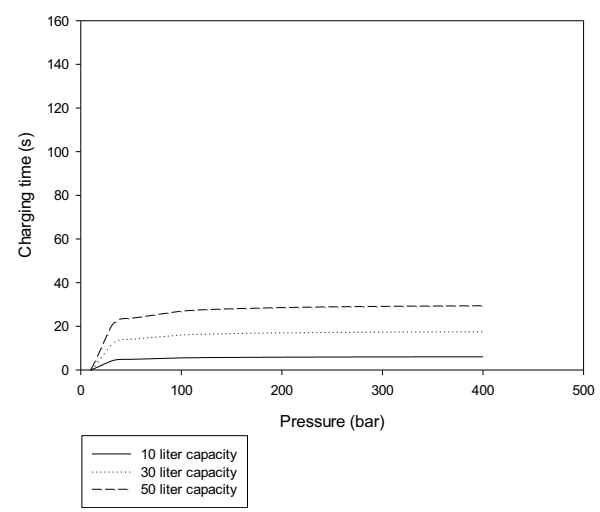

(b)

Fig. 11. Charging time for $\mathrm{V}_{\mathrm{d}}=10 \mathrm{~cm}^{3} / \mathrm{rev}$ (a) and $\mathrm{V}_{\mathrm{d}}=50 \mathrm{~cm}^{3} / \mathrm{rev}$ (b) at pre-charge pressure of 10 bar.

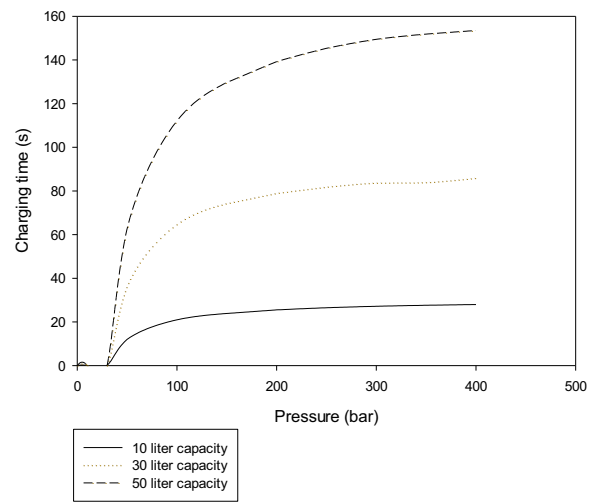

(a)

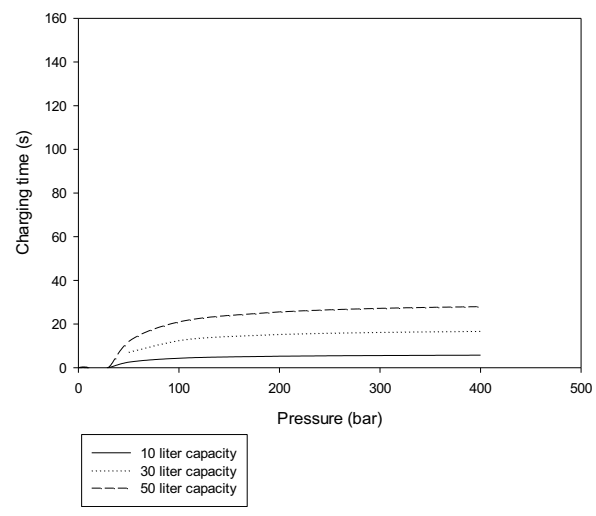

(b)

Fig. 12. Charging time for $V_{d}=10 \mathrm{~cm}^{3} / \mathrm{rev}$ (a) and $\mathrm{V}_{\mathrm{d}}=50 \mathrm{~cm}^{3} / \mathrm{rev}$ (b) at pre-charge pressure of 30 bar. 


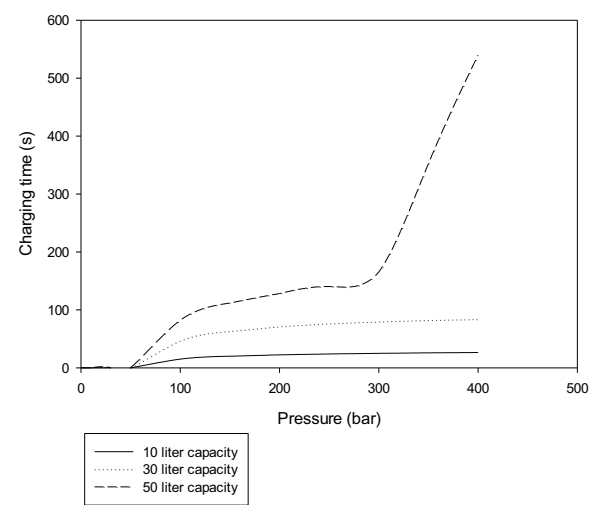

(a)

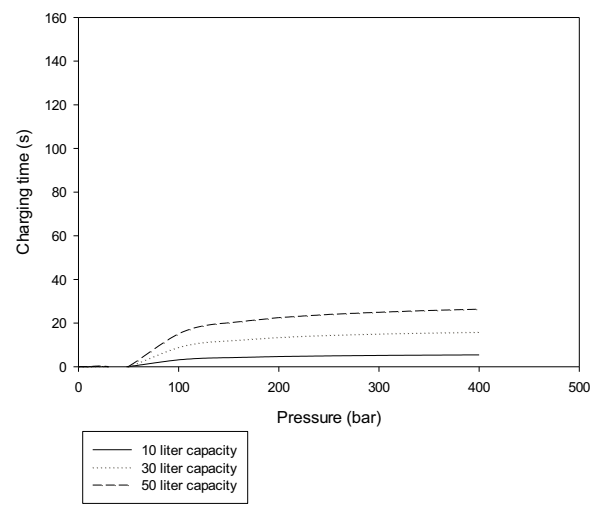

(b)

Fig. 13. Charging time for $\mathrm{V}_{\mathrm{d}}=10 \mathrm{~cm}^{3} / \mathrm{rev}$ (a) and $\mathrm{V}_{\mathrm{d}}=50 \mathrm{~cm}^{3} / \mathrm{rev}$ (b) at pre-charge pressure of 50 bar.

\section{Conclusions}

In conclusion, the simulation result has managed to show some of the critical parameters which affect the performance of charging process. Effective volume relationship has shown that volume displacement does not influence the level of volume in accumulator instead pre-charge value do. The increase in pre-charge value gives adverse effect to the effective volume. Effect of temperature witnessed that the higher the volume displacement, the lower the heat in the system. In most cases, the larger the storage capacity, the higher the resulting temperature. However, this effect can be reduced by raising the volume displacement. In this context, pre-charge doesn't give significant effect to the temperature. The effect of charging time has shown that the higher the volume displacement causes the increasing of time required for charging. However, the size of the storage capacity has an adverse effect on the charging time. One thing to avoid in this process is the combination of low volume displacement, high storage capacity, and high pre-charge pressure because it gave severe impact to the charging time in pressure elevation around 300 to 400 bar. Charging time increased from 7 to 21 times greater than the 10 -litre storage, $10 \mathrm{~cm}^{3} / \mathrm{rev}$ displacement, and 50 bar pre-charge setting.

This paper was made possible by a scholarship from the Ministry of Higher Education and Universiti Teknikal Malaysia Melaka. The authors also would like to be obliged to Universiti Malaysia Pahang for giving the opportunities and providing facilities to complete this study.

\section{References}

1. K. D. Huang, S. C. Tzeng, Appl. Energy, 80, 47 (2005)

2. J. Cobb, March 2016 Dashboard, http://www.hybridcars.com/, 11-May-2016

3. US HEV sales by model, http://www.afdc.energy.gov/data/10301. 11-May-2016

4. A. Boretti, J. Stecki, SAE Commer. Veh. Eng. Congr., (2012)

5. T. Lin, Q. Wang, B. Hu, W. Gong, Automation in Construction, 19, 11 (2010)

6. P. Mrdja, N. Miljic, S. J. Popovic, M. Kitanovic, V. Petrovic, Green Design Conference, 129 (2012)

7. W. Backé, Proc. Inst. Mech. Eng., 207, 193 (1993) 
8. A. Boretti, S. Zanforlin, SAE Int., (2014)

9. H. Holloway, Peugeot 2008 Hybrid Air prototype first drive review, http://www.autocar.co.uk/car-review/peugeot/2008/first-drives/peugeot-2008-hybridair-prototype-first-drive-review, 30-Jan-2015

10. U. Diego-ayala, An investigation into hybrid power trains for vehicles with regenerative braking, (2007)

11. Z. Dimitrova, P. Lourdais, F. Mar, Energy, 86, 574 (2015)

12. F. Tavares, R. Johri, Z. Filipi, J. of Eng. Gas Turbines Power, 133, no. 12, (2011)

13. F. Tavares, Thermally Boosted Concept for Improved Energy Storage Capacity of a Hydro - Pneumatic Accumulator, (2011)

14. Accumulators, https://www.boschrexroth.com/en/us, 11-May-2016

15. M. P. Lammert, J. Burton, P. Sindler, A. Duran, SAE Int. J., 4, no. 1, (2014)

16. R. P. Kepner, SAE Tech. Pap., (2002)

17. Automation Studio, http://www.automationstudio.com/, 11-May-2016

18. Automation Studio Target for Simulink, http://www.brautomation.com/en/products/software/automation-studio-target-for-simulink/, 11-May2016

19. J. Ma, H. Schock, U. Carlson, A. Hoglund, M. Hedman, SAE Tech. Pap. (2006)

20. J. Zhang, C. Lv, J. Gou, D. Kong, Proc. Inst. Mech. Eng., 226, 1289 (2012)

21. P. Achten, G. Vael, M. I. Sokar, T. Kohmäscher, Proc. JFPS, 47 (2008)

22. A. Esposito, Fluid Power with Applications, (2013)

23. Bladder Accumulators, http://www.parker.com/portal/site/, 11-May-2016

24. P. Puddu, M. Paderi, Energy, 57, 326 (2013) 